\title{
How good is the Prevent model for estimating the health benefits of prevention?
}

\author{
Henrik Brønnum-Hansen
}

\begin{abstract}
Study objective-Prevent is a public health model for estimating the effect on mortality of changes in exposure to risk factors. When the model is tested by simulating a development that has already taken place, the results may differ considerably from the actual situation. The purpose of this study is to test the Prevent model by applying it to a synthetic cohort in which the development is unaffected by concealed factors.

Design-A micro-simulation model was developed to create basic data for Prevent and give "exact" results as to changes in risk factor prevalences and mortality. The estimates of Prevent simulations were compared with the "exact" results.

Main results-Modelling one risk factor related to a cause specific mortality gave fairly similar results by the two methods. Including two risk factors Prevent tends slightly to overestimate the health benefits of prevention.

Conclusions-The differences between the "exact" mortality and the Prevent estimates will be small for realistic scenarios and Prevent provide reasonable estimates of the health benefits of prevention. (F Epidemiol Community Health 1999;53:300-305)
\end{abstract}

The simulation model Prevent estimates the effect on mortality in a population of changes in risk factor prevalence resulting from trends and interventions. ${ }^{1}$ The model takes the multifactorial nature of the associations between risk factors and diseases, time dimensions, and demography into account. Prevent is intended to be used by policy makers. Thus, the model demands only limited data and a reasonable use gives intelligible results founded on well established and biologically plausible theory.

Prevent is one of several models that have been developed to simulate the effect of disease prevention or health promotion programmes. The Framingham Heart Study has provided the basis for a simple method for predicting coronary heart disease based on equations combining risk factor prevalences and estimates of associations between risk factors and disease. ${ }^{2}{ }^{3}$ The Coronary Heart Policy Model is a risk factor model that relies on the Framingham data. ${ }^{4}$

A weakness of a general use of the Framingham equations is that one cannot be sure that these are valid for other populations because parameter effects associated with the Framingham cohort may be different and may have changed over time. Furthermore, different choices of risk factors are limited.

In Canada a POpulation HEalth Model (POHEM) was developed to meet health statistical and policy needs. The model is comprehensive with various applications. ${ }^{67}$ One weakness of POHEM and other microsimulation models is that large data are needed and the models are difficult to implement.

The risk factor model building methodologies are diverse. Fundamentally different are macro-simulation models (like Prevent) based on aggregated data compared with microsimulation models (like POHEM) that act at the individual level. Especially microsimulation models are often designed in such detail that data for appropriate use of the models are not available. Therefore these models will probably not be widely distributed as effective tools for potential users. For this reason it is important for policy making that users of simple models like Prevent can trust the results.

An obvious way of testing Prevent is to check whether it is capable of describing an evolution that has already taken place. The problem is that observed secular trends in mortality cannot be explained by actual knowledge of risk factor distributions and effects. The purpose of this study is to test the Prevent model on a synthetic population to see if it does what it is supposed to do. The synthetic population is established by a micro-simulation model. To make sure that the micro-simulation makes sense it is tested by a validated statistical technique, Cox regression.

\section{Methods}

THE PREVENT MODEL

The Prevent model was developed to predict the effect on mortality in a population of changes in exposure to one or more risk factors. Model simulations are based on aggregated data and describe changes in total mortality and mortality resulting from selected causes of death.

The model is based on the epidemiological effect measure, the "potential impact fraction", which is a multifactorial generalisation of the aetiological fraction. This fraction is a measure of the reduction in the proportion of new cases of disease in a population after a change in exposure to the associated risk factors.

The model allows one risk factor to be associated with more than one disease, and one disease to be associated with more than one risk factor. The simulations take into account the fact that an increased risk for disease will not be reduced immediately as exposure stops 


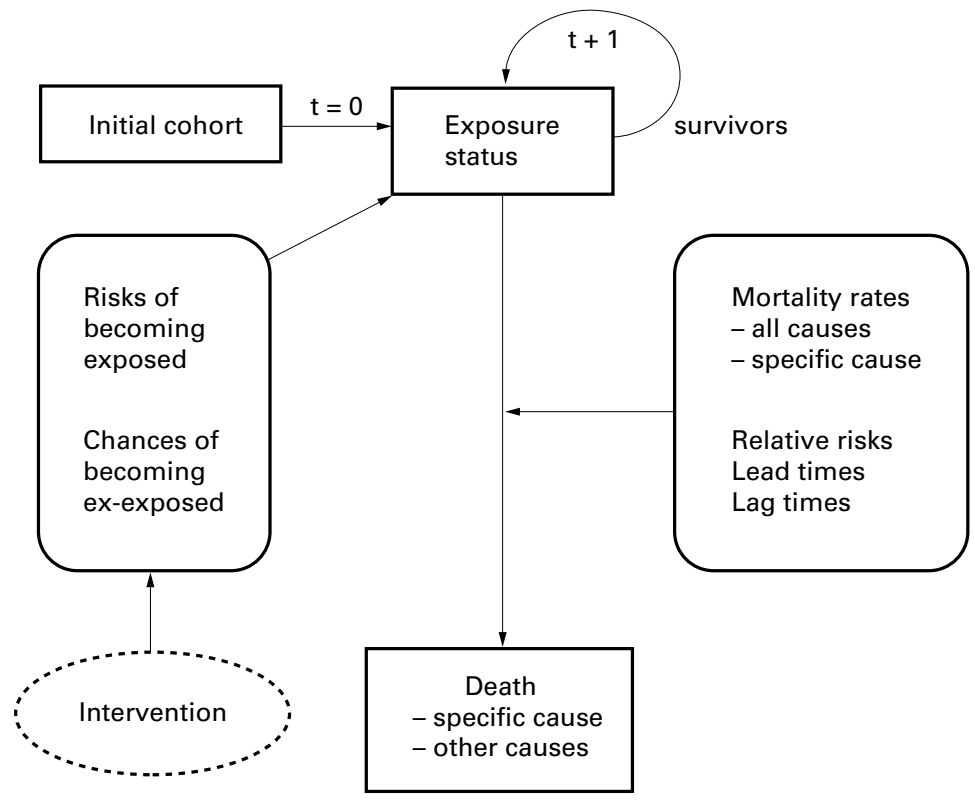

Figure 1 Diagram of micro-stimulation.

but will be reduced gradually over time (lag time). Demographic evolution is also taken into account in the simulation. The calculations are based on successive solutions to difference equations expressing the evolution year by year during the simulation period.

The model operates with the size of the population and total and relevant cause specific death probabilities for the year in which the simulation starts, the prevalence proportions for exposure to risk factors, and trends in this respect. Furthermore, relative risks and lag times are incorporated.

The result of a simulation is the evolution of mortality in two populations: (1) the reference population, which reflects the demographic changes and autonomous trends in exposure to the risk factors, and (2) the intervention population, which is also affected by reduced exposure to the risk factors. As a result, several descriptions of the mortality in the two populations are given, including mortality rates, survival curves, and life expectancy, together with measures of differences between the two populations.

The Prevent model is described in detail in a dissertation published in $1989 .^{1}$ The basic methodology can be found in appendix 1 .

THE MICRO-SIMULATION METHOD

At the start of simulation of a population, the people are each given an age. The future life story of each person is then generated by exposing it year by year to a risk of death, which is divided into a specific cause of death and (all) other causes. These risks of death are derived from suitably selected total and cause specific death rates. The specific cause of death is associated with risk factors. If a person has been exposed to a risk factor, the cause specific death risk is increased in accordance with a given relative risk. The risk may increase gradually over a certain number of years after the start of exposure (lead time). During the time that the person is exposed to the risk fac- tor, there is a chance that the exposure will stop and the person will then be an ex-exposed. During passage into this state, the previously increased (cause specific) risk of death will be gradually reduced (lag time) to a level between never having been exposed and being exposed. It is assumed that an ex-exposed person remains ex-exposed-that is, the death risk remains at the ex-exposed level. All transitions between states of exposure and eventual death are based on comparisons of risks and random numbers between 0 and 1 drawn from a random number generator. A diagram of the model is shown in figure 1 .

After a certain number of years, the person will die, the maximum age having been fixed at 110 years. Whether the person is alive, exposed, or ex-exposed to the risk factors has now been determined for each year after the beginning of simulation until death - that is, we have a synthetic population with data on individual historical events. The microsimulation calculations were carried out by means of the SAS software system. ${ }^{89}$ Further details of the methodology can be found in appendix 2 .

\section{Checking the result of the micro-simulation}

A certain number of years after the beginning of simulation, each person may be described as being or having been exposed to the risk factors or not. As the deaths of the people have been recorded, survival analyses may be carried out to determine the effect of the risk factors on cause-specific and total mortality.

If data on possible changes in exposures to risk factors in the course of the follow up period are used, time dependent covariates in a Cox regression model may be defined. ${ }^{10}{ }^{11}$ In this way it is possible to check whether the simulation has developed satisfactorily, as the estimates of relative risks must correspond to the selected values in the simulation.

\section{TESTING THE PREVENT MODEL ON A SYNTHETIC} COHORT

A synthetic birth cohort is created by simulation as described above. The evolution of mortality in the cohort depends on the initially selected rates of death from the specific cause and all causes of death and on changes in exposure to the risk factors and the selected relative risks. As the cohort is being "followed" until all are dead, mortality can be estimated. The risk factor prevalences of the simulated cohort may also be described year by year. If the simulation is repeated with new risks of exposure, the mortality will be different. In particular, this simulation will give other prevalences of the risk factors. These prevalence figures will then be used in a Prevent simulation in which the cohort created first defines the reference population of the Prevent model, and the new cohort will be represented in Prevent as the intervention population. This will make it possible to study the similarity of the results for changes in mortality in the two models. 
Table 1 Proportions of exposed and ex-exposed in simulated cohorts (one risk factor)

\begin{tabular}{llllll}
\hline & \multicolumn{2}{l}{ Reference population } & & \multicolumn{2}{c}{ Intervention population } \\
\cline { 2 - 3 } Age group $(y)$ & Exposed (\%) & Ex-exposed (\%) & & Exposed (\%) & Ex-exposed (\%) \\
\hline $30-39$ & 30.8 & 17.9 & 27.7 & 20.9 \\
$40-49$ & 28.0 & 22.4 & & 0.0 & 50.4 \\
$50-59$ & 25.7 & 24.6 & & 0.0 & 50.3 \\
$60-69$ & 24.7 & 24.9 & & 0.0 & 50.1 \\
$70-79$ & 22.9 & 25.3 & & 0.0 & 50.0 \\
$80-89$ & 20.9 & 25.8 & & 0.0 & 49.9 \\
\hline
\end{tabular}

Material

The artificial cohort was established on the basis of mortality rates for Danish men in the period 1986-90. Lung cancer was chosen as the specific cause of death; the risk factors could be cigarette smoking and air pollution. Only in the first test, however, does the scenario reflect some kind of reality, cigarette smoking being the only risk factor. The disease and risk factors are of no significance for the actual purpose, and neither is the choice of parameter values.

For the simulations, a cohort of $400000-9$ year old boys, 4000 of each age, was chosen.

Simulations provide estimates of the mortality of the entire cohort for use as basic data in the Prevent model. Other basic data for determining the reference population for the Prevent model are also provided by the simulation. In particular, prevalence figures for the exposure of the reference population to the risk factors are determined. To test the model, another set of probabilities is chosen, thus obtaining other prevalence figures for exposure and ex-exposure. These prevalence figures are the basis for a Prevent simulation of the effect of an intervention.

After 30 years, the surviving boys will be 30-39 year old men. These men constitute the start population in the Prevent model, which then simulates the evolution 50 years onwards, up to the ages of 80-89.

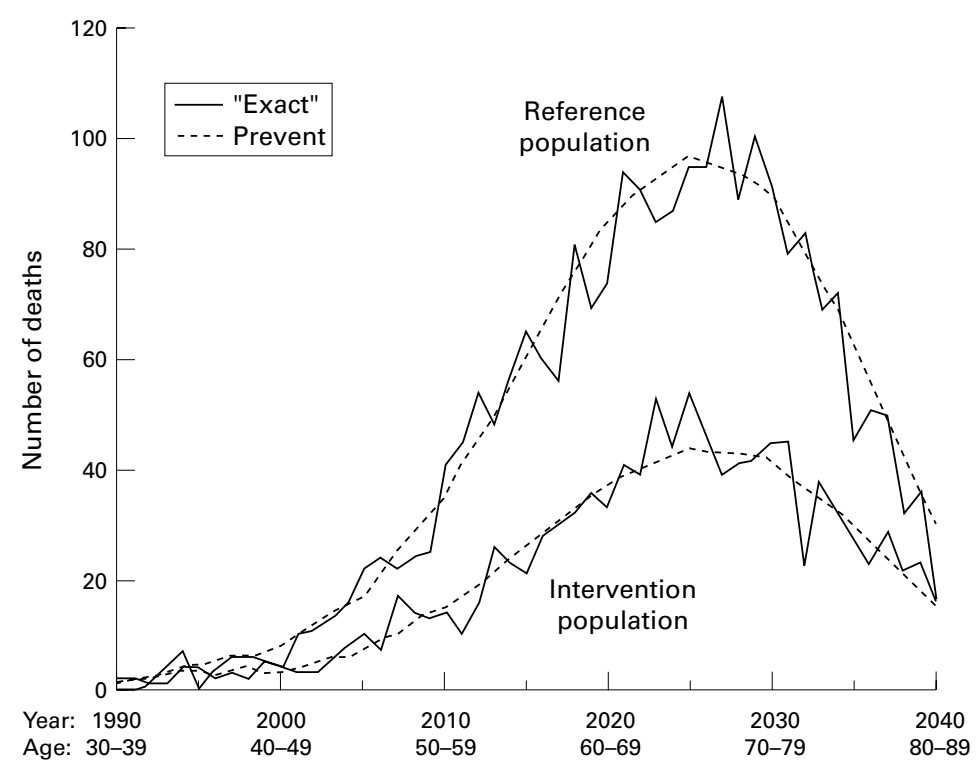

Figure 2 Number of cause specific deaths in the simulated cohorts ("exact") and based on the Prevent simulation for each year during 1990-2040. First test.

\section{Results}

FIRST TEST (ONE RISK FACTOR)

Two sets of probabilities of exposure and ex-exposure were selected, one defining the reference population and one defining the intervention population. The probabilities for the intervention population represent a radical change in risk: that nobody over 40 years of age is exposed.

Table 1 shows the risk factor prevalences for the simulated cohorts. Note that the prevalence figures for different ages represent the same cohort growing up and followed up over a period of 50 years.

The following parameter values for the association between the risk factor and the specific cause of death were chosen: relative risk for exposed, 10; lead time, 10 years; relative risk for ex-exposed, 2; lag time, 10 years.

To check whether the simulation has produced a cohort in which the cause specific mortality is related to the risk factor according to the chosen relative risk values, a Cox regression model was defined, and relative risks with $95 \%$ confidence intervals estimated. In this model, the survival of each person is followed up for 90 years, and time dependent covariates for exposed and ex-exposed are included. The relative risk estimates were 10.5 $(9.4,11.8)$ for exposed and $2.2(1.9,2.5)$ for ex-exposed in the reference population. In the intervention population, the number of exposed people dying of the specific cause was too small to estimate the relative risk for the exposed people; for the ex-exposed in the intervention population, the estimate was 2.0 $(1.8,2.3)$. Thus, there is good agreement between the selected and estimated relative risks, and the simulations are satisfactory in this respect.

In the cohort corresponding to the reference population, 31666 people died in the course of 50 years, and 2328 of these died from the specific cause; according to the Prevent model, 31711 are dead, and 2402 died from the specific cause. In the intervention population, 31440 died, 1097 from the specific cause; the Prevent model estimates are 31265 dead, 1088 from the specific cause.

Figure 2 shows the evolution in cause specific mortality in the two populations. As can be seen, there is close similarity between the results of the two methods. The large fluctuations in the "exact" figures for the simulated cohorts are because 40000 people are in fact a small number, especially for a relatively rare cause of death. What is decisive is that the curves follow the same pattern throughout the process.

SECOND TEST (TWO RISK FACTORS)

In the following test, two risk factors were included. To obtain results that demonstrate visible differences in mortality with the two methods, exposure probabilities giving high prevalence figures were chosen. Therefore, high relative risks were also selected. These choices are unlikely to reflect real life. 
Table 2 Proportions of exposed and ex-exposed in simulated cohorts (two risk factors)

\begin{tabular}{|c|c|c|c|c|c|c|c|c|}
\hline \multirow{3}{*}{$\begin{array}{l}\text { Age group } \\
\text { (y) }\end{array}$} & \multicolumn{4}{|c|}{ Reference population } & \multicolumn{4}{|c|}{ Intervention population } \\
\hline & \multicolumn{2}{|c|}{ risk factor $1(\%)$} & \multicolumn{2}{|c|}{ risk factor $2(\%)$} & \multicolumn{2}{|c|}{ risk factor $1(\%)$} & \multicolumn{2}{|c|}{ risk factor $2(\%)$} \\
\hline & Exposed & Ex-exposed & Exposed & Ex-exposed & Exposed & Ex-exposed & Exposed & Ex-exposed \\
\hline $30-39$ & 30.9 & 17.0 & 45.7 & 0.0 & 30.8 & 17.6 & 45.8 & 0.0 \\
\hline $40-49$ & 28.1 & 21.9 & 50.0 & 8.2 & 17.4 & 33.0 & 22.0 & 36.1 \\
\hline $50-59$ & 24.3 & 24.4 & 41.5 & 22.4 & 5.9 & 44.0 & 4.9 & 59.4 \\
\hline $60-69$ & 19.8 & 25.3 & 27.9 & 33.7 & 1.8 & 47.2 & 0.6 & 63.4 \\
\hline $70-79$ & 15.3 & 25.7 & 17.6 & 39.6 & 0.5 & 47.2 & 0.1 & 62.6 \\
\hline $80-89$ & 12.5 & 24.8 & 11.1 & 42.6 & 0.1 & 46.0 & 0.0 & 61.7 \\
\hline
\end{tabular}

Table 2 shows the prevalences of the risk factors for the simulated cohorts.

The following parameter values for the associations between the risk factors and the specific cause of death were chosen:

risk factor 1 :

- relative risk for exposed, 10; lead time, 10 years

- relative risk for ex-exposed, 2; lag time, 10 years.

risk factor 2 :

- relative risk for exposed, 15; lead time, 2 years

- relative risk for ex-exposed, 5; lag time, 2 years.

The agreement between the chosen and estimated values (by Cox regression) of the relative risks was again good. The estimated relative risks for the reference population were as follows: exposed to risk factor 1: 9.5 (9.0, 10.0); ex-exposed to risk factor 1: 2.0 (1.9, 2.1); exposed to risk factor $2: 15.0(13.9,16.1)$; ex-exposed to risk factor $2: 5.2(4.8,5.6)$. For the intervention population, the estimates were: exposed to risk factor 1: $9.8(8.9,10.9)$; ex-exposed to risk factor $1: 2.3(2.1,2.4)$; exposed to risk factor 2: $13.6(11.7,15.7)$; ex-exposed to risk factor 2: $4.8(4.3,5.3)$.

In the reference population, 33779 people died in the course of 50 years, 9413 of these from the specific cause; according to the Prevent model, 33747 died, 9603 from the specific cause. In the intervention population,

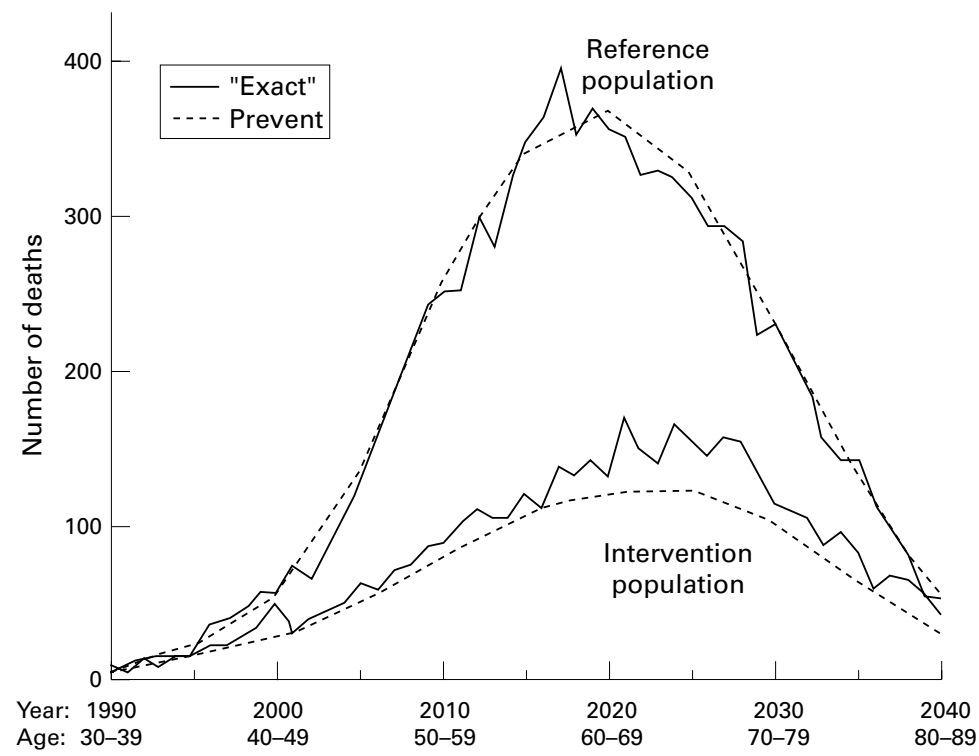

Figure 3 Number of cause specific deaths in the simulated cohorts ("exact") and based on the Prevent simulation for each year during 1990-2040. Second test.
32246 died, 4335 from the specific cause; the Prevent model estimates are 32010 dead, 3599 from the specific cause.

Figure 3 shows the cause specific mortality in the two populations. It can be seen that the Prevent model systematically underestimates the mortality for the intervention population. This problem is discussed below.

\section{Discussion}

Increased computer capacity has significantly improved the possibilities for using large, complex simulation and prognosis models and for developing tools aimed at a wide circle of users. The Prevent model is a simulation model developed for use by policy makers at both the national and local levels. The model is easy to use and does not require an unreasonable amount of data.

Users of the Prevent and other models naturally wish to trust the results. Confidence in a model may be strengthened by a number of tests, the obvious one being to examine whether the model can "predict" a development that has already taken place. But as a model is always a simplification of reality, historical tests often show deviations between the actual development and the predictions of the model.

It is well known from other subject areas that prognoses fail. The problem is recognised, for instance, in demography, for example, population forecasts and fertility projections, and in econometric forecasts. Prognoses seldom prove to be correct for systems that change by complex interactions of many factors, especially if the preconditions on which the prognosis is based are not fulfilled. If those preconditions cannot be verified, however, it may be difficult to establish why a prognosis is not correct.

A test that could solve this problem is one that evaluates the model with a development that is unaffected by concealed factors. In this study, the Prevent model was tested by applying it to a synthetic population generated by micro-simulation.

The first test involved one risk factor only, and the second test included two risk factors. Good agreement was achieved between the "actual" mortality in the synthetic population and the mortality estimated by the Prevent model, both as regards the total number of deaths and the cause specific mortality for the reference population. This is to be expected, as the data used in the Prevent model were derived directly from the simulated cohort. The real test comes when the mortality for the intervention population obtained by application of the two 
methods is compared, as the calculations in the two methods are basically different. The intervention population in the Prevent model is defined exclusively by means of data derived from the simulated cohort, and the prevalence figures found when the artificial cohort is again simulated with new probabilities for exposure and ex-exposure. The calculations in the simulated cohort take place at the individual level, whereas the calculations in the Prevent model are based on aggregated data.

In the micro-simulation, the cause specific death risks for the exposed and ex-exposed people are established by multiplying the cause specific mortality for the unexposed people by the relative risks. The high risk groups will have increased risk of cause specific mortality. Thus, as the cohort ages, the exposed persons will die relatively fast, and the mortality among the survivors will be dominated by that of the unexposed and ex-exposed people. In other words, selective mortality is taken into account in simulating mortality at the individual level.

The calculations in the Prevent model are based on cause specific death risks for the entire cohort, obtained by combining mortality, risk factor prevalences, and relative risks. As the potential impact fraction represents the reduction in proportional mortality applied to 10 year age groups, the selective mortality of high risk groups will not be reflected in a reduction in the prevalence of exposure within age groups as the cohort grows older. This explains why the Prevent model tends to slightly overestimate the cause specific mortality in the reference population, whereas mortality is underestimated in the intervention population.

The problem of selective mortality would be reduced in the Prevent model if more than six age groups were allowed as input for the prevalence of exposed people. Such data will, however, seldom be available; furthermore, the problem will present itself almost only in simulations of very dramatic reductions in the prevalence of exposure and when the absolute mortality differential is high. A third test (not shown) incorporating the effect of interaction by increasing the specific cause of death for the simultaneous effect of two risk factors was also carried out with the purpose of studying the consequence of ignoring such an effect. The result was a further deviation between the exact and estimated mortality for the intervention population. However, the interaction effect would only be of real significance if the exposure prevalences and the interaction relative risk are high. Furthermore, the problem of interaction is virtually impossible to take into account in practice, owing to the lack of cross tabulated data on the prevalence of exposure to risk factors and estimates of interaction relative risks.

The rates of mortality caused by ischaemic heart disease have declined in many countries over the past $15-20$ years. It is often claimed that this decline is because of a healthier lifestyle, with reduced tobacco smoking, more people exercising, reduced dietary fat, fewer overweight people, and fewer people suffering from hypertension. It is evident that the Prevent model could be used to verify this
KEY POINTS

- Prevent is a public health model for predicting the effect on mortality of changes in exposure to risk factors.

- Because of unknown factors Prevent cannot be evaluated by simulating a development that has already taken place. But the model can be tested by applying it to a synthetic population with no "hidden" factors.

- Although Prevent tends slightly to overestimate the health benefits of prevention the estimates are reasonable for realistic scenarios of health promotion.

claim. For this purpose, historical data on mortality rates and on changes in the prevalence of the risk factors must be available, and this was the case in a Danish study. ${ }^{12}$ The Prevent model was unable to predict the drastic decline in mortality attributable to ischaemic heart disease on the basis of changes in smoking, hypertension, hypercholesterolaemia, and alcohol consumption. The total change in exposure to these four risk factors shows, however, only a slightly favourable tendency. A similar result was found in a Swedish study ( $M$ Stenbeck, personal communication).

The fact that the Prevent model could not predict the decline in mortality attributable to ischaemic heart disease in Denmark and Sweden does not imply that it failed. When studying the evolution of mortality from ischaemic heart disease in the 1980s it is important to take into account the improved survival after myocardial infarct brought about by thrombolytic therapy and other improvements in treatment. The Prevent model takes risk factors into account but not the effects of new and better treatment procedures. Other explanations for the poor predictivity may be that the data on prevalence and the relative risk estimates were unreliable or incomplete, especially for older people. Unreliable lag time estimates may also contribute to a bad fit in the model. The information on smoking may have been incomplete: for instance, information is required about the age at start of smoking and the duration of smoking. Other risk factors should be included, such as environment, working conditions, and socioeconomic factors, and interactions between risk factors should be taken into account. Moreover, there may be cohort effects on mortality. Finally, coding of causes of death often changes over time.

The conclusion to be derived from these tests is that the Prevent model tends to overestimate the health benefits of prevention because the estimates of mortality are a little too high in the reference population and too low in the intervention population. Actual health promotion activities would usually be far more modest than those simulated here, and the overestimate would be a minor source of error.

I am grateful to Professor Louise Gunning-Schepers (University of Amsterdam, Institute of Social Medicine) and Deputy Director Mette Madsen of the Danish Institute for Clinical Epidemitor Mette Madsen of the Danish Institute for
ology for fruitful discussions and comments. 
Funding: this study was funded by the Danish Ministry of Health grant for research and analysis and the sectorial research grant.

Conflicts of interest: none.

Appendix 1

BASIC METHODOLOGY OF THE PREVENT MODEL

Let the proportion of exposed people be $\mathrm{P}_{\mathrm{t}-L A \mathrm{~T}^{\mathrm{r}, \mathrm{n}} \mathrm{z}, \mathrm{i}}^{\mathrm{r}, \mathrm{i}, \mathrm{s}}$

and let $R^{\mathrm{r}, \mathrm{r}, \mathrm{s}, \mathrm{A}, \mathrm{n}, \mathrm{i}}$ signify the relative risk

where the indices are:

$\mathrm{t}$ for time

$\mathrm{r}$ for risk factor

$j=0,1$ for reference $(0)$ or intervention

population (1)

$\mathrm{s}$ for sex

A for age

$\mathrm{n}$ for exposure category

$i$ for ex-exposure level

$\mathrm{z}$ for disease

and LAT is latency time.

The trend impact fraction, TIF, and the potential impact fraction, PIF, are defined as

$\mathrm{TIF}_{\mathrm{t}}^{\mathrm{r}, \mathrm{z}, \mathrm{s}, \mathrm{A}}=\frac{\mathrm{PRR}_{0}^{\mathrm{r}, \mathrm{j}=0, \mathrm{z}, \mathrm{s}, \mathrm{A}}-\mathrm{PRR}_{\mathrm{t}}^{\mathrm{r}, \mathrm{j}=0, \mathrm{z}, \mathrm{s}, \mathrm{A}}}{\mathrm{PRR}_{0}^{\mathrm{r}, \mathrm{j}=0, \mathrm{z}, \mathrm{s}, \mathrm{A}}}$
$\mathrm{PIF}_{\mathrm{t}}^{\mathrm{r}, \mathrm{z}, \mathrm{s}, \mathrm{A}}=\frac{\mathrm{PRR}_{\mathrm{t}}^{\mathrm{r}, \mathrm{j}=0, \mathrm{z}, \mathrm{s}, \mathrm{A} A}-\mathrm{PRR}_{\mathrm{t}}^{\mathrm{r}, \mathrm{j}=1, \mathrm{z}, \mathrm{s}, \mathrm{A}}}{\mathrm{PRR}_{\mathrm{t}}^{\mathrm{r}, \mathrm{j}=0, \mathrm{z}, \mathrm{s}, \mathrm{A}}}$

where

$\operatorname{PRR}_{\mathrm{t}}^{\mathrm{r}, \mathrm{j}, \mathrm{z}, \mathrm{s}, \mathrm{A}}=\sum_{\mathrm{n}=1}^{\mathrm{cn}} \sum_{\mathrm{i}=0}^{\mathrm{ID}} \mathrm{P}_{\mathrm{t}-\mathrm{LAT}}^{\mathrm{r}, \mathrm{j}, \mathrm{s}, \mathrm{A}, \mathrm{n}, \mathrm{i}, \mathrm{i}, \mathrm{R}} \mathrm{RR}^{\mathrm{r}, \mathrm{z}, \mathrm{s}, \mathrm{A}, \mathrm{n}, \mathrm{i}, \mathrm{i}}$

$\mathrm{cn}$ is the total number of exposure categories and ID is the total number of ex-exposure levels.

For the specific disease, $\mathrm{z}$, associated with $\mathrm{rf}$ risk factors

$$
\begin{aligned}
& \mathrm{TIF}_{\mathrm{t}}^{\mathrm{z}, \mathrm{s}, \mathrm{A}}=1-\prod_{\mathrm{r}=1}^{\mathrm{rf}}\left(1-\mathrm{TIF}_{\mathrm{t}}^{\mathrm{r}, \mathrm{z}, \mathrm{s}, \mathrm{A}}\right) \\
& \mathrm{PIF}_{\mathrm{t}}^{\mathrm{z}, \mathrm{s}, \mathrm{A}}=1-\prod_{\mathrm{r}=1}^{\mathrm{rf}}\left(1-\mathrm{PIF}_{\mathrm{t}}^{\mathrm{r}, \mathrm{z}, \mathrm{s}, \mathrm{A}}\right)
\end{aligned}
$$

Let $\mathrm{M}^{\mathrm{s}, \mathrm{A}}$ and $\mathrm{M}^{\mathrm{z}, \mathrm{s}, \mathrm{A}}$ signify the baseline total and cause specific mortality rates and let zt be total number of diseases. The simulated mortality rates for the reference $(j=0)$ and the intervention population $(j=1)$ are

$$
\begin{aligned}
& M_{t}^{j=0, s, A}=M^{s, A}-\sum_{z=1}^{z t} T_{t}^{z, s, A} M_{t}^{z, s, A} \\
& M_{t}^{j=1, s, A}=M^{s, A} \\
& -\sum_{z=1}^{z t}\left[1-\left(1-\operatorname{TIF}_{t}^{z, s, A}\right)\left(1-P_{t}^{z, s, A}\right)\right] M^{z, s, A}
\end{aligned}
$$

\section{Appendix 2}

DETAILS FOR THE MICRO-SIMULATION METHOD Each person goes through a course of life that ends with death. The person who initially is unexposed to any given risk factor may

- remain unexposed until his or her death

- pass from an unexposed state to an exposed state

- pass from an exposed state to an exexposed state.
For each person at any time (or age) t, two random numbers $r$ and $s$ between 0 and 1 are drawn. Let $i$ signify the combined exposure category and $\mathrm{p}_{\mathrm{i}}(\mathrm{t})$ the probability of dying from the specific cause for a person with exposure category $i$ and let $q(t)$ signify the probability of dying from other causes. The person dies at time $t$ from the specific cause if $r<p_{i}(t)$ and from other causes if $\mathrm{s}<\mathrm{q}(\mathrm{t})$.

As long as the person is alive it is determined for any risk factor-also at any time (or age) whether or not an unexposed person becomes exposed or whether an exposed person becomes ex-exposed, by evaluating one year risks of this transition as compared with numbers drawn at random.

The cause specific death risks are derived from the initially selected death rates and are adjusted according to exposure: For risk factor $j$ let $R R_{i 1}$ and $R R_{22}$ be the relative risks for exposed and ex-exposed persons respectively, and let $1_{j 1}$ and $1_{j_{2}}$ be lead and lag times. When a person becomes exposed to risk factor $j$ the relative risk will increase gradually (by linear interpolation) until the exposure stops or the value reaches $\mathrm{RR}_{\mathrm{i} 1}$ after $\mathrm{l}_{\mathrm{i} 1}$ years. If an exposed person becomes ex-exposed to risk factor $\mathrm{j}$ before the relative risk has exceeded the value $R_{\mathrm{i}_{2}}$ the relative risk is fixed at the actual level. If the person becomes ex-exposed after the value $R_{\mathrm{i}_{2}}$ is reached the relative risk will be gradually decreased (by linear interpolation) until the level $R_{\mathrm{j}_{2}}$ is reached after max. $1_{\mathrm{i} 2}$ years.

The probability $\mathrm{p}_{\mathrm{i}}(\mathrm{t})$ of dying from the specific cause is determined by multiplying the appropriate relative risks according to the risk factor levels with the death probability for unexposed people.

When the person dies, the following variables are recorded: age of death, length of life span, the cause of death, and possible previous passing from different risk factor exposure states.

1 Gunning-Schepers LJ. The health benefits of prevention: a simulation approach. Health Policy 1989;12:1-256.

2 Wilson PWF, Castelli WP, Kannel WB. Coronary risk prediction in adults (The Framingham Heart Study). $A m \mathcal{F}$ Cardiol 1987;59:91-4G.

3 Anderson KM, Wilson PWF, Odell PM, et al. An updated coronary risk profile. A statement for health professionals. Circulation 1991;83:356-62.

4 Weinstein MC, Coxson PG, Williams LW, et al. Forecasting coronary heart disease incidence, mortality, and cost: The coronary heart disease incidence, mortality, and cost: The
Coronary Heart Disease Policy Model. Am f Public Health Coronary Heart Dised

1987;77:1417-26.
5 Hunink MG, Goldman L, Tosteson AN, et al. The recent decline in mortality from coronary heart disease, 19801990. The effect of secular trends in risk factors and treatment. $\mathcal{F} A M A$ 1997;277:535-42.

6 Wolfson MC. POHEM - a framework for understanding and modelling the health of human populations. World Health Stat $Q$ 1994;47:157-76.

7 Evans WK, Will BP, Berthelot JM, et al. Estimating the cost of lung cancer diagnosis and treatment in Canada: The of lung cancer diagnosis and treatment in Can model. Can f Oncol 1995;5:408-19.

8 SAS Institute Inc. SAS language. Reference, version 6, 1st ed. Cary, NC: SAS Institute Inc, 1990:1042.

9 SAS Institute Inc. SAS procedures guide. Version 6. 3rd ed. Cary, NC: SAS Institute Inc, 1990:705

10 SAS Institute Inc. SAS technical report P-217. SAS/STAT Software: The PHREG Procedure. Version 6. Cary, NC: SAS Institute Inc, 1991:59.

11 Kalbfleisch JD, Prentice RL. The statistical analysis of failure time data. New York: Wiley, 1980:321.

12 Brønnum-Hansen H, Siøl A. Forudsigelse af udviklingen i dødelighed af iskæmisk hjertesygdom i Danmark 1982dødelighed af iskæmisk hjertesygdom i Danmark $1982-$ 991 ved anvendelse af simulationsmodellen Prevent [Pre1982-1991 by use of the simulation model "Prevent"]. Ugeskr Laeger 1996;158:4898-904. 